\title{
On Open Problems of Nonnegative Inverse Eigenvalues Problem*
}

\author{
Junliang Wu \\ College of Mathematics and Statistics of Chongqing University, Chongqing, China \\ E-mail: jlwu678@tom.com \\ Received March 15, 2011; revised June 19, 2011; accepted June 25, 2011
}

\begin{abstract}
In this paper, we give solvability conditions for three open problems of nonnegative inverse eigenvalues problem (NIEP) which were left hanging in the air up to seventy years. It will offer effective ways to judge an NIEP whether is solvable.
\end{abstract}

Keywords: Inverse Eigenvalues Problem, Nonnegative Inverse Eigenvalues Problem, Solvability, Nonnegative Matrix, Spectrum of Matrix

\section{Introduction}

In 1937, Kolmogorov [1] asked the question: When is a given complex number an eigenvalues of some (entry-wise) nonnegative matrix? The answer is: Every complex number is an eigenvalue of some nonnegative matrix [2]. Suleimanova [3] extended Kolmogorov's question in 1949 to the following well known problems which are called the nonnegative inverse eigenvaluese problems (NIEP) (also see [4]).

Problem 1 (NIEP). Determine necessary and sufficient conditions for a set of $n$ complex numbers to be the eigenvalues of a nonnegative matrix of order $n$.

Problem 1 is open for $n \geq 4$. The case $n=2$ is easy while then $n=3$ is due to Loewy and London [5] (R.Loewy and D.D.London, A note on an inverse problem for nonnegative matrices, Linear and Multilinear algebra, 6 (1978), 83-90).

In the same paper [3] Suleimanova also considered the following real nonnegative inverse eigenvalues problem and gave a sufficient condition.

Problem 2 (RNIEP). Determine necessary and sufficient conditions for a set of $n$ real number to be the eigenvalues of a nonnegative matrix of order $n$.

Problem 2 is open for $n \geq 5$. Fiedler [6] posed the following symmetric nonnegative inverse eigenvalues problem in 1974.

Problem 3 (SNIEP). Determine necessary and suffi-

${ }^{*}$ This work is supported by national natural science foundations of China (Grant No. 70872123). cient conditions for a set of $n$ real number to be the eigenvalues of a symmetric nonnegative matrix of order $n$.

Throughout the article, $R$ denotes set of real numbers, $C$ denotes set of complex numbers.

Problem 1, Problem 2 and Problem 3 have not been solved yet since they were presented. To find the solvability of these three problems, people have been studying them in the recent 70 years (refer to the references), the achievements people have got and their limitations and practical application depict were evaluated in scholarly treatise [7] (Moody T. Chu, Gene H. Golub, Inverse Eigenvalue Problems: Theory, Algorithms, and Applications, Oxford University press. P 93-122) and article [8] (Ricardo L.SoTo, Reliability by symmetric nonnegative matrces, http://www.scielo.cl/pdf/proy/v24n1/art06. pdf). Readers also may refer to [9-26] for some previous results. In some articles, some necessary conditions and sufficient conditions for the three problems above have been given under some small dimension or special cases [7]. Also See the survey paper [26] and the book [2, Chapter VII]. The earliest study on the subject of NIEP was perhaps due to the Russian mathematician Suleimanova (1949) on stochastic matrices, followed by Perfect $(1953,1955)$. The first systematic treatment of eigenvalues of symmetric nonnegative matrices can probably be attributed to Fiedler (1974). A more comprehensive study was conducted by Boyle and Handelman (1991) using the notion of symbolic dynamics to characterize the conditions under which a given set is a portion of the spectrum of a nonnegative matrix or primitive matrix. 
General treatises on nonnegative matrices and applications include the classics by Berman and Plemmons (1979) and Minc (1988). Both books devote extensive discussion to the NIEPs as well.

As [7, p94] said, most of the discussions in the literature center around finding conditions to qualify a given set of values as the spectrum of some nonnegative matrices. A short list of references giving various necessary or sufficient conditions includesBarrett and Johnson (1984), Boyle and Handelman (1991), Friedland (1978), Friedland and Melkman (1979), Loewy and London (1978), de Oliveira (1983), and Reams (1996). The difficulty is that the necessary condition is usually too general and the sufficient condition too specific. Under a few special sufficient conditions, the nonnegative matrices can be constructed numerically (Soules, 1983).

In this paper, we will use a general method to give the solvability conditions of Problem 1 and Problem 2, we also discuss problem 3 and pose some new topics which caused by the Problem 3. Our approach is quite straightforward, but it offers an effective way to judge whether an NIEP is solvable.

To facilitate discuss the solvability condition of NIEP, we will integrate NIEP with IEP, is the following problem 4. Firstly we will solve problem 4, and then with some additional conditions, the solvability conditions of Problem 1 and Problem 2 can be give accordingly.

Problem 4. (Inverse eigenvalue problem-IEP) Given a list of complex numbers

$\Lambda=\left\{\lambda_{1}, \lambda_{2}, \cdots, \lambda_{n}\right\}$, investigate whether there is a $n \times n$ real matrix with spectrum $\Lambda$ and how to determine such a matrix.

\section{Basic Requirement of the Existence of the Solutions to IEP and NIEP}

For any a given list of numbers $\Lambda=\left\{\lambda_{1}, \lambda_{2}, \cdots, \lambda_{n}\right\}$, if it have odd number elements which are pure complex numbers (a complex number $a+b i$ is said to be a pure complex if $b \neq 0$ ) or even number pure complex numbers with at least a complex number's conjugation are not in the list, we can assert that any $n \times n$ real matrix can not satisfy the demand of Problem 1-4. Because any real matrix's spectrum always depends on a real coefficient polynomial, but the complex roots of a real coefficient polynomial always comes in pairs.

It means that for the existence of the matrices which present in problem $1-4$, the list $\Lambda=\left\{\lambda_{1}, \lambda_{2}, \cdots, \lambda_{n}\right\}$ must appear as follows:

$$
\Lambda=\left\{r_{1}, r_{2}, \cdots, r_{k}, z_{1}, z_{2}, \cdots, z_{l}, \bar{z}_{1}, \bar{z}_{2}, \cdots, \bar{z}_{l}\right\}
$$

where $r_{i} \in R(0 \leq i \leq k, 0 \leq k \leq n), \quad z_{j} \in C, \bar{z}_{j}$ is conjugation of $z_{j} \quad(0 \leq j \leq l, 0 \leq l \leq n), k+2 l=n$. When $k=0$, it means that all of $\lambda_{1}, \lambda_{2}, \cdots, \lambda_{n}$ are pure complex numbers. When $l=0$, it means that all of $\lambda_{1}, \lambda_{2}, \cdots, \lambda_{n}$ are real numbers. That is, $\Lambda=\left\{\lambda_{1}, \lambda_{2}, \cdots, \lambda_{n}\right\}$ must be closed under complex conjugation, the closed conception has been presented by some articles (see [3], p. 476, [7], p. 93).

Next, we will begin our work with a basic result related to Problem 4.

\section{An Answer to the IEP}

Compared with the NIEP, the IEP-inverse eigenvalue problem seems to be easier one, so we discuss it firstly.

Theorem 3.1. For a given list of complex numbers $\Lambda=\left\{\lambda_{1}, \lambda_{2}, \cdots, \lambda_{n}\right\}$, if it has closed property under complex conjugation, there must be at least one real matrix $A$ with spectrum $\Lambda$.

Proof. Since $\Lambda=\left\{\lambda_{1}, \lambda_{2}, \cdots, \lambda_{n}\right\}$ has closed property under complex conjugation, it has form (2.1). So, we can use it to construct the following polynomial:

$$
f(x)=\prod_{i=1}^{n}\left(x-\lambda_{i}\right)
$$

We note that (3.1) is a symmetry real coefficient polynomial. By expanding, merging similar items and simplifying, we suppose (3.1) becomes

$$
f(x)=x^{n}+a_{1} x^{n-1}+\cdots+a_{n-1} x+a_{n}
$$

Then $a_{1}, a_{2}, \cdots, a_{n}$ are real numbers and (3.2) has roots $\lambda_{1}, \lambda_{2}, \cdots, \lambda_{n}$.

We use $a_{1}, a_{2}, \cdots, a_{n}$ to construct companion matrix of (3.2):

$$
\boldsymbol{A}=\left[\begin{array}{cccccc}
0 & 1 & 0 & \cdots & 0 & 0 \\
0 & 0 & 1 & 0 & \cdots & 0 \\
\cdots & \cdots & \cdots & \cdots & \cdots & \cdots \\
0 & 0 & 0 & \cdots & 0 & 1 \\
-a_{n} & -a_{n-1} & -a_{n-2} & \cdots & -a_{2} & -a_{1}
\end{array}\right]
$$

It is not difficult to test that the characteristic polynomial of $\boldsymbol{A}$ is (3.2) and (3.1) exactly. It means that $\boldsymbol{A}$ has spectrum $\Lambda=\left\{\lambda_{1}, \lambda_{2}, \cdots, \lambda_{n}\right\}$.

Thus, we can draw the conclusion. The proof is complete.

We note that using $n$ given numbers to determine $n \times n$ unknown variables (a $n \times n$ matrix) maybe a problem of indeterminate equations, which shows that 
there may be the relation of many to one between the two of $n \times n$ unknown variables and $n$ given numbers. That is, the solution of IEP and its form may not be unique. In fact, we have the following theorem:

Theorem 3.2. For a given list of complex numbers $\Lambda=\left\{\lambda_{1}, \lambda_{2}, \cdots, \lambda_{n}\right\}$, if it has closed property under complex conjugation and there exist $n \times n$ invertible real matrix $\boldsymbol{P}$ makes $\boldsymbol{P}^{-1} \boldsymbol{A P}=\boldsymbol{B}$, then $\boldsymbol{B}$ also has spectrum $\Lambda=\left\{\lambda_{1}, \lambda_{2}, \cdots, \lambda_{n}\right\}$, where $\boldsymbol{A}$ is the matrix which is constructed in the proof of Theorem 3.1.

\section{Solvability Condition of Problem 1 and Problem 2}

In this section we will discuss the key problem that is the solvability condition of Problem 1 and Problem 2 of NIEP. We note that the difference between IEP and NIEP is that, NIEP needs people to find at least one nonnegative matrix with a given list of complex numbers $\Lambda=\left\{\lambda_{1}, \lambda_{2}, \cdots, \lambda_{n}\right\}$ as its spectrum rather than others. Based on this requirement, we give the following Theorem:

Theorem 4.1. For a given list of complex numbers $\Lambda=\left\{\lambda_{1}, \lambda_{2}, \cdots, \lambda_{n}\right\}$, if it has closed property under complex conjugation, then the sufficient condition that has at least one nonnegative matrix $\boldsymbol{A}$ with spectrum $\Lambda$ is that

$$
\left\{\begin{array}{c}
\lambda_{1}+\lambda_{2}+\cdots+\lambda_{n} \geq 0 \\
\lambda_{1} \lambda_{2}+\lambda_{1} \lambda_{3}+\cdots+\lambda_{n-1} \lambda_{n}=\sum_{\substack{i, j=1 \\
i<j}}^{n} \lambda_{i} \lambda_{j} \leq 0 \\
\lambda_{1} \lambda_{2} \lambda_{3}+\lambda_{1} \lambda_{2} \lambda_{4}+\cdots+\lambda_{n-2} \lambda_{n-1} \lambda_{n}=\sum_{\substack{i, j, k=1 \\
i<j<k}}^{n} \lambda_{i} \lambda_{j} \lambda_{k} \geq 0 \\
\cdots \cdots \\
\lambda_{1} \lambda_{2} \cdots \lambda_{n} \begin{cases}\geq 0 & n \text { is a odd number } \\
\leq 0 & n \text { is a even number }\end{cases}
\end{array}\right.
$$

Proof. It is similar to prove the IEP in Theorem 3.1, we use $\Lambda=\left\{\lambda_{1}, \lambda_{2}, \cdots, \lambda_{n}\right\}$ to construct the following symmetry real coefficient polynomial:

$$
f(x)=\prod_{i=1}^{n}\left(x-\lambda_{i}\right)
$$

By expanding and merging similar items, we suppose (4.2) becomes

$$
f(x)=x^{n}+a_{1} x^{n-1}+\cdots+a_{n-1} x+a_{n}
$$

then $a_{1}, a_{2}, \cdots, a_{n}$ are real numbers and (4.3) has roots $\lambda_{1}, \lambda_{2}, \cdots, \lambda_{n}$.

According to an algebra basic theorem of real coefficient polynomial, we can get the following equalities:

$$
\left\{\begin{array}{l}
\lambda_{1}+\lambda_{2}+, \cdots,+\lambda_{n}=-a_{1} \\
\lambda_{1} \lambda_{2}+\lambda_{1} \lambda_{3}+\cdots+\lambda_{n-1} \lambda_{n}=\sum_{\substack{i, j=1 \\
i<j}}^{n} \lambda_{i} \lambda_{j}=a_{2} \\
\lambda_{1} \lambda_{2} \lambda_{3}+\lambda_{1} \lambda_{2} \lambda_{4}+\cdots+\lambda_{n-2} \lambda_{n-1} \lambda_{n}=\sum_{\substack{i, j, k=1 \\
i<j<k}}^{n} \lambda_{i} \lambda_{j} \lambda_{k}=-a_{3} \\
\quad \cdots \\
\lambda_{1} \lambda_{2} \cdots \lambda_{n}=(-1)^{n} a_{n}
\end{array}\right.
$$

It is clear that if (4.1) holds, we can deduce that $-a_{1} \geq 0,-a_{2} \geq 0, \cdots,-a_{n} \geq 0$.

If we use $a_{1}, a_{2}, \cdots, a_{n}$ to construct real matrix $\boldsymbol{A}$ as (3.3), $\boldsymbol{A}$ is a nonnegative matrix and its characteristic polynomial is the same with (4.3) and (4.2). That is, A has spectrum

$$
\Lambda=\left\{\lambda_{1}, \lambda_{2}, \cdots, \lambda_{n}\right\} .
$$

Thus, the proof is complete.

Obviously, Theorem 4.1 gives a solvability condition of Problem 1.

In theorem 4.1, the verifying terms are simpler and easy to be actualized.

For instance, if we let

$$
\lambda_{1}=-1+\frac{1}{2} i, \lambda_{2}=-1-\frac{1}{2} i, \lambda_{3}=3, \lambda_{4}=-1
$$

we have

$$
\begin{aligned}
& \lambda_{1}+\lambda_{2}+\lambda_{3}+\lambda_{4}=0 \geq 0 \\
& \lambda_{1} \lambda_{2}+\lambda_{1} \lambda_{3}+\lambda_{1} \lambda_{4}+\lambda_{2} \lambda_{3}+\lambda_{2} \lambda_{4}+\lambda_{3} \lambda_{4}=-\frac{23}{4} \leq 0 \\
& \lambda_{1} \lambda_{2} \lambda_{3}+\lambda_{1} \lambda_{2} \lambda_{4}+\lambda_{1} \lambda_{3} \lambda_{4}+\lambda_{2} \lambda_{3} \lambda_{4}=\frac{17}{2} \geq 0 \\
& \lambda_{1} \lambda_{2} \lambda_{3} \lambda_{4}=-\frac{15}{2} \leq 0 \\
& \text { It is clear that } \boldsymbol{A}=\left[\begin{array}{cccc}
0 & 1 & 0 & 0 \\
0 & 0 & 1 & 0 \\
0 & 0 & 0 & 1 \\
\frac{15}{4} & \frac{17}{2} & \frac{23}{4} & 0
\end{array}\right] \text { is a nonnegative }
\end{aligned}
$$

matrix and it has spectrum

$$
\Lambda=\left\{-1+\frac{1}{2} i,-1-\frac{1}{2} i, 3,-1\right\}
$$


Theorem 4.1 shows that for a given list of complex numbers $\Lambda=\left\{\lambda_{1}, \lambda_{2}, \cdots, \lambda_{n}\right\}$, people can construct a nonnegative matrix through the sufficient condition above and make this matrix have spectrum $\Lambda=\left\{\lambda_{1}, \lambda_{2}, \cdots, \lambda_{n}\right\}$.

It is obvious that if $\lambda_{1}, \lambda_{2}, \cdots, \lambda_{n}$ is a given list of $n$ real numbers, it is the special case of list of complex numbers $\Lambda=\left\{\lambda_{1}, \lambda_{2}, \cdots, \lambda_{n}\right\}$, so we have the following Theorem:

Theorem 4.2. For any a given list of real numbers $\Lambda=\left\{\lambda_{1}, \lambda_{2}, \cdots, \lambda_{n}\right\}$, the sufficient condition that has at least one nonnegative matrix $A$ with spectrum $\Lambda$ is that

$$
\left\{\begin{array}{l}
\lambda_{1}+\lambda_{2}+\cdots+\lambda_{n} \geq 0 \\
\lambda_{1} \lambda_{2}+\lambda_{1} \lambda_{3}+\cdots+\lambda_{n-1} \lambda_{n}=\sum_{\substack{i, j=1 \\
i<j}}^{n} \lambda_{i} \lambda_{j} \leq 0 \\
\lambda_{1} \lambda_{2} \lambda_{3}+\lambda_{1} \lambda_{2} \lambda_{4}+\cdots+\lambda_{n-2} \lambda_{n-1} \lambda_{n}=\sum_{\substack{i, j, k=1 \\
i<j<k}}^{n} \lambda_{i} \lambda_{j} \lambda_{k} \geq 0 \\
\cdots \\
\lambda_{1} \lambda_{2} \cdots \lambda_{n} \begin{cases}\geq 0 & n \text { is a odd number } \\
\leq 0 & n \text { is a even number }\end{cases}
\end{array}\right.
$$

The proof of Theorem 4.2 can refer to Theorem 4.1, so it is omitted.

Theorem 4.2 gives a solvability condition of Problem 2.

Theorem 4.3. For a given list of complex numbers $\Lambda=\left\{\lambda_{1}, \lambda_{2}, \cdots, \lambda_{n}\right\}$, if it has closed property under complex conjugation and there exist $n \times n$ invertible real matrix $\boldsymbol{P}$ makes $\boldsymbol{P}^{-1} \boldsymbol{A P}=\boldsymbol{B}$ and $\boldsymbol{B}$ is nonnegative matrix, $\boldsymbol{B}$ also has spectrum $\Lambda$, where $\boldsymbol{A}$ is the matrix which is constructed in the proof of Theorem 4.1.

Theorem 4.3 also shows that the solution to NIEP may not be unique.

Next, we will discuss Problem 3.

According to Theorem 4.2 and Theorem 4.3, we know that as long as $\Lambda=\left\{\lambda_{1}, \lambda_{2}, \cdots, \lambda_{n}\right\}$ satisfy (4.1), then there must be at least one nonnegative matrix $A$ has spectrum $\Lambda=\left\{\lambda_{1}, \lambda_{2}, \cdots, \lambda_{n}\right\}$. Further, if there exist $n \times n$ invertible real matrix $\boldsymbol{P}$ makes $\boldsymbol{P}^{-1} \boldsymbol{A P}=\boldsymbol{B}$ and $B$ is nonnegative symmetric matrix, $\boldsymbol{B}$ also has spectrum $\Lambda$, where $\boldsymbol{A}$ is the matrix which is constructed in the proof of Theorem 4.1. If that were true, a solvability condition of Problem 3 would be give. The question is that it needs people to prove the following three new topics of matrix theory further:

1) For any a given $n \times n$ nonnegative matrix $\boldsymbol{A}$, whether there must be an $n \times n$ invertible real matrix $\boldsymbol{P}$ makes $\boldsymbol{P}^{-1} \boldsymbol{A P}=\boldsymbol{B}$ and also makes $\boldsymbol{B}$ to be a nonnegative matrix?

2) For any a given $n \times n$ nonnegative matrix $\boldsymbol{A}$, whether there must be an $n \times n$ invertible real matrix $\boldsymbol{P}$ makes $\boldsymbol{P}^{-1} \boldsymbol{A P}=\boldsymbol{B}$ and also makes $\boldsymbol{B}$ to be a symmetric matrix?

3) For any a given $n \times n$ nonnegative matrix $\boldsymbol{A}$, whether there must be an $n \times n$ invertible real matrix $\boldsymbol{P}$ makes $\boldsymbol{P}^{-1} \boldsymbol{A P}=\boldsymbol{B}$ and also makes $\boldsymbol{B}$ a both nonnegative matrix and symmetric matrix;

From the evidence we have heard so far, there are no existing conclusions for the above problems. But we conjecture each of three new propositions above is holds. In fact, since $\boldsymbol{A}$ is a known nonnegative matrix, in numerous real matrices, there must be invertible real matrix $\boldsymbol{P}$ makes $\boldsymbol{P}^{-1} \boldsymbol{A P}=\boldsymbol{B}$ and $\boldsymbol{B}$ a both nonnegative matrix and symmetric matrix.

To avoid adrift from the subject of this paper, we leaves the question to readers first.

\section{References}

[1] A. N. Kolmogorov, "Markov Chains with Countably Many Possible States,” Bull University, Moscow, 1937, pp. 1-16.

[2] H. Minc, "Nonnegative Matrices,” John Wiley and Sons, New York, 1988, p. 166.

[3] K. R. Suleimanova, "Stochastic Matrices with Real Eigenvalues,” Soviet Mathematics Doklady, Vol. 66, 1949, pp. 343-345.

[4] M. Fiedler, "Eigenvalues of Nonnegative Symmetric Matrices," Linear Algebra Applied, Vol. 9, 1974, pp. 119-142. doi:10.1016/0024-3795(74)90031-7.

[5] M. T. Chu and G. H. Golub, "Inverse Eigenvalue Problems: Theory, Algorithms, and Applications,” Oxford University, Oxford, pp. 93-122.

[6] R. L. SoTo, "Reliability by Symmetric Nonnegative Matrices,” http://www.scielo.cl/pdf/proy/v24n1/art06.pdf.

[7] A. Borobia, "On the Nonnegative Eigenvalue Problem," Linear Algebra Applied, Vol. 223-224, 1995, pp. 131-140. doi:10.1016/0024-3795(94)00343-C.

[8] M. Boyle and D. Handelman, "The Spectra of Nonnegative Matrices via Symbolic Dynamics,” Annals of Mathematics, Vol. 133, 1991, pp. 249-316. doi:10.2307/2944339

[9] X. Z. Zhan, "Matrix Theory,” Academic Press, Chinese, 2008, p. 127.

[10] P. Egleston, "Nonnegative Matrices with Prescribed Spectra,” Dissertation, Central Michigan University, 2001.

[11] C. Johnson, "Row Stochastic Matrices Similar to Doubly Stochastic Matrices," Linear and Multilinear Algebra, Vol. 10, No. 2, 1981, pp. 113-130. doi:10.1080/03081088108817402

[12] C. Johnson, T. J. Laffey and R. Loewy, "The Real and the Symmetric Nonnegative Inverse Eigenvalue Problems are Different," Proceedings of the American Mathematical Society, Vol. 124, 1996, pp. 3647-3651. doi:10.1090/S0002-9939-96-03587-3

[13] F. Karpelevich, "On the Eigenvalues of a Matrix with 
Nonnegative Elements," Izvestia Akademii Nauk SSSR Metally, Vol. 15, 1951, pp. 361-383.

[14] R. B. Kellogg, "Matrices Similar to a Positive or Essentially Positive Matrix," Linear Algebra Applied, Vol. 4, No. 3, 1971, pp. 191-204. doi:10.1016/0024-3795(71)90015-2

[15] C. Knudsen and J. J. McDonald, "A Note on THE Convexity of the Realizable set of Eigenvalues for Nonnegative Symmetric Matrices," Electronic Journal Linear Algebra, Vol. 8, 2001, pp. 110-114.

[16] T. Laffey, "Realizing Matrices in the Nonnegative Inverse Eigenvalue Problem,” Texts in Mathematics ,Series B, University, Coimbra, 1999, pp. 21-32.

[17] T. Laffey and E. Meehan, “A refinement of an inequality of Johnson, Loewy, and London on Nonnegative Matrices and Some Applications," Electron Journal Linear Algebra, Vol. 3, 1998, pp. 119-128.

[18] T. Laffey and E. Meehan, "A Characterization of Trace zero Nonnegative 5×5 Matrices,” Linear Algebra Applied, Vol. 302-303, No. 1, 1999, pp. 295-302. doi:10.1016/S0024-3795(99)00099-3

[19] J. J. McDonald and M. Neumann, “The Soules Approach to the Inverse Eigenvalue Problem for Nonnegative Symmetric Matrices of Order n-5," Contemporary Mathematics, Vol. 259, 2000, pp. 387-407.
[20] L. Mirsky and H. Perfect, "Spectral Properties of Doubly Stochastic Matrices,” Mathematics and Statistics, Vol. 69 No. 1, 1965, pp. 35-57. doi:10.1007/BF01313442

[21] H. Perfect, "Methods of Constructing Certain Stochastic Matrices," Duke Mathematical Journal, Vol. 20, No. 3, 1953, pp. 395-404. doi:10.1215/S0012-7094-53-02040-7

[22] N. Radwan, "An Inverse Eigenvalue Problem for Symmetric and Normal Matrices,” Linear Algebra Applied, Vol. 248, No. 15, 1996, pp. 101-109. doi:10.1016/0024-3795(95)00162-X

[23] R. Reams, "An Inequality for Nonnegative Matrices and the Inverse Eigenvalue Problem," Linear and Multilinear Algebra, Vol. 41, No. 4, 1996, pp. 367-375. doi:10.1080/03081089608818485

[24] G. Wuwen, "Eigenvalues of Nonnegative Matrices," Linear Algebra Applied, Vol. 266, No. 15 1997, pp. 261-270. doi:10.1016/S0024-3795(96)00007-9

[25] R. Loewy and D. London, “A Note on an Inverse Problem for Nonnegative Matrices," Linear and Multilinear Algebra, Vol. 6, No. 1, 1978, pp. 83-90. doi:10.1080/03081087808817226.

[26] P. D. Egleston and T. D. Lenker, "Sivaram K. Narayan, the Nonnegative Inverse Eigenvalue Problem," Linear Algebra and Its Applications, Vol. 379, No. 1, 2004, pp. 475-490. 